\title{
Tumor Profiling-Directed Precision Cancer Therapy - Comparison of Commercial and Academic Clinical Utility
}

Jaak Janssens $^{1^{*}}$, William M. Gallagher ${ }^{2}$, Andrew Dean ${ }^{3}$, Giovanni Ussia ${ }^{4}$ and Gordon Stamp ${ }^{5}$

${ }^{1}$ Department of Oncology, Limburg Oncology Center, Belgium

${ }^{2} U C D$ School of Biomolecular and Biomedical Science, UCD Conway Institute, University College Dublin, Belfield, Dublin 4, Ireland

${ }^{3}$ St John of God Hospital, Subiaco, WA 6008, Australia

${ }^{4}$ Dipartimento di Medicina e Chirurgia, OspedaleSant'Orsola, Universita di Bologna, Via Massarenti 9, 40138 Bologna, Italia

${ }^{5}$ Imperial College Section of Investigative Medicine, Hammersmith Campus, London, United Kingdom

\section{Abstract}

Molecular tumor profiling for patients with advanced or recurrent solid tumors is increasingly adopted as standard of care in oncology, as it has been demonstrated that improved clinical outcomes can result from selection of the optimal therapy for individual patients.

The Clinical Utility of a molecular profiling approach must demonstrate whether they lead to a reconsideration of the treatment plan and whether this improves the clinical outcome in profiled patients. Our comparison of 4 commercially available molecular profiling services and those of purely academic approaches, revealed a wide range of impact on treatment choice and clinical benefit. These variations in profiling service performance highlight the need for ongoing justification, quality control and evaluation if this methodology is to be effectively deployed within the clinical setting. It also means that a profiling approach without clinical outcome data can only be considered to perform in line with the lowest performing comparator in terms of clinical utility and cost effectiveness until otherwise demonstrated.
Publication History:

Received: August 24, 2017

Accepted: October 05, 2017

Published: October 07, 2017

\section{Keywords:}

Clinical benefit, Clinical utility, Cost-effectiveness, Impact on treatment decision, Tumor profiling

\section{Introduction}

The promise of precision oncology is to deliver key information and have gained based on molecular specific alterations to individual cancer patients in the hope of finding a tailored treatment solution for their disease. Initial large scale attempts to profile patients in the hope of directing them to molecularly matched clinical trials have highlighted that only a small proportion of patients have actionable alterations which are suitable for enrollment into a trial. In 2,000 patients profiled at M.D. Anderson, of whom $789(39 \%)$ had at least one alteration in potentially actionable genes, only $83(4.2 \%)$ were enrolled in a matched clinical trial [1]. The use of a comprehensive assay, MSKIMPACT, to prospectively sequence 10,000 patients with diverse cancers at Memorial Sloan Kettering found that, despite 37\% of tested tumors harboring a clinically relevant alteration, only 527 (11\%) of the first 5,009 patients profiled were enrolled onto genomically matched clinical trials [2]. The initial experiences have left some oncologists to express frustration at the relatively few patients to have benefited from precision oncology, and there is some debate on whether this is due to the approach in general or reflects the methodology used, tumor types or availability of appropriate drugs $[3,4]$. Tumor sequencing is already used as standard of care to guide routine treatment decisions in many cancers, but the basic tenet of precision medicine is that this needs to have broad application across all solid cancers [5]. This has lead to the development of large basket trials such as the National Cancer Institute's Molecular Analysis for Therapy Choice (NCI-MATCH) and Targeted Agent and Profiling Utilization Registry (TAPUR) to look at the outcomes of matched therapy. For oncologists who are actively integrating tumor profiling into their patient's care today, it can be challenging to understand the differences in clinical utility and benefit between numerous available molecular profiling services. The often-considerable costs at the patient's expense require justification. Limited data exist about the clinical outcomes achieved with different profiling solutions. Molecular tumor profiling utilizes a panel of biomarkers that may influence therapy selection for an individual patient. This may involve single or combined technologies including
Next-Generation Sequencing (NGS), Immunohistochemistry (IHC), Polymerase Chain Reaction (PCR) or In Situ Hybridization (ISH). A handful of biomarkers have strong associations with treatment outcomes and gained regulatory approval as "Companion Diagnostics". Others have a less validated association better suited for patients for whom no well-documented standard of care exists. Limited data exist on the use of predictive biomarkers and the respective therapies for which utility was established in one cancer type, in different cancer types. A few examples, like the use of trastuzumab for NSCLC with activating HER2 mutations, have been included in clinical guidelines based on limited clinical data, but as knowledge increases, we will see other examples emerge from research in clinical trials or serendipitous case reports.

The validity of such predictive biomarker results is crucial since oncologists often prescribe drugs with major side effects based on the results. It is inadvisable to blindly trust the results of a molecular profile, as many factors may influence therapeutic response.

All services now have adapted to work with formalin fixed paraffin embedded tissue specimens, which is the routine method of pathological analysis used by diagnostic laboratories worldwide. Despite this, it is remarkable that it is a relatively uncontrolled process in a clinical setting (e.g. devitalization and fixation time variations, differing reagents and processing schedules etc.), underlining the

"Corresponding Author: Prof. Jaak Janssens, University Hasselt, Klein Hilststraat 5, 3500 Hasselt, Belgium Tel: + 3211 275734, Fax: + 3211 255334; E-mail: jaak.janssens@ecprevention.org

Citation: Janssens J, Gallagher WM, Dean A, Ussia G, Stamp G (2017) Tumor Profiling-Directed Precision Cancer Therapy - Comparison of Commercial and Academic Clinical Utility. Int J Surg Surgical Porced 2: 123. doi: https://doi. org/10.15344/2456-4443/2017/123

Copyright: () 2017 Janssens et al. This is an open-access article distributed under the terms of the Creative Commons Attribution License, which permits unrestricted use, distribution, and reproduction in any medium, provided the original author and source are credited. 
Citation: Janssens J, Gallagher WM, Dean A, Ussia G, Stamp G (2017) Tumor Profiling-Directed Precision Cancer Therapy - Comparison of Commercial and Academic Clinical Utility. Int J Surg Surgical Porced 2: 123. doi: https://doi.org/10.15344/2456-4443/2017/123

Page 2 of 10

need for comprehensive and rigorous quality control to understand which biomarkers can be analyzed reproducibly and more importantly which cannot (e.g. phosphorylated proteins). The leading professional molecular profile services include several layers of quality checks and controls, which are almost impossible to implement in a smaller service with less volume and expert resources.

The development of less invasive technologies such as profiling circulating free DNA or tumor cells might be initially more appealing to patients, however, these technologies are still highly experimental and certainly not validated for clinical decision-making. We have therefore excluded these methodologies from this review.

Key differences between molecular profiling services lie in the range and scope of platforms used to assess biomarkers. Whereas currently approved companion diagnostics would require the use of several technologies for their assessment (e.g. IHC, ISH \& NGS for HER2 analysis) those are neither established nor validated in some laboratories. Importantly, while NGS is able to detect single nucleotide variants (SNVs), insertions and deletions (InDels), gene amplifications, and some fusions, it is not able to detect or reliably predict transcriptional regulation or changes in protein expression. This becomes a limitation if protein expression is related to the drug effect.

The published medical scientific data about drug biomarker associations is extensive and requires frequent updating of biomolecular panels to ensure optimal relevance. This is best accomplished by an expert multidisciplinary team that shares its understanding with the oncologist to support therapy decisions, either via direct communication or by developing algorithms that generate a concise qualitative and quantitative report with potential therapeutic options, that is shared with the treating oncologist. The interpretation of the profiling results for the treating oncologist is essential in determining the ultimate treatment decision and thus optimize outcome for each particular patient.

It is imperative that selection of cancer therapy in patients at late stages is derived from an evidence base and can demonstrate a positive clinical benefit versus toxic side effects ratio to justify continued investment from Governmental or insurance medical care providers $[6,7]$.

The true value of prospective tumor profiling is measured by its ability to influence treatment decisions and improve outcomes for patients. In this paper, we aim to compare and contrast the clinical utility of 4 commercial approaches, along with those from leading academic institutions to show that not all approaches to precision medicine are the same in terms of quality and cost-effectiveness. This research can be the basis for inclusion of newer decision algorithms in cancer patients.

\section{Impact on Treatment Choice}

We compared the treatment choice impact of 4 commercial services in this paper - Caris Molecular Intelligence ${ }^{\infty}$ from Caris Life Sciences, FoundationOne $e^{\circ}$ from Foundation medicine, $\mathrm{PCDx}^{\mathrm{Tm}}$ from Paradigmdx, and OncoDEEP ${ }^{\text {ix }}$ from OncoDNA to independent academic clinical trials by analyzing published clinical data [8-55]. As the services are offered for all solid tumors, and the actual treatment choice itself reflects the real-world situation and not theoretical actionable alterations, all prospective and retrospective studies were considered without differentiation on country, prior treatments or tumor type. CMI has the highest impact on treatment choice (77\%), followed by $\mathrm{PCDx}^{\mathrm{mm}}(26 \%)$, and FoundationOne $(19 \%)$ (Figure $1 \mathrm{~A}$ and Table 1). The impact on treatment choice of profiling services that have been developed by academic institutions in house is similar to that of FoundationOne ${ }^{\infty}$. For OncoDEEP ${ }^{\mathrm{rx}}$, we were unable to find any published references that demonstrated impact on treatment choice or corresponding clinical benefit in a patient population, so no clinical utility for this profiling service can be documented.

\section{Clinical Benefit}

Clinical benefit can be defined as a positive impact on patient health as defined by the treating physician and varies between the clinical studies of incorporating profiling approaches into clinical practice. Achieving clinical benefit from molecular profiling-guided treatments depends on several critical factors that all need to be considered: the inclusion of appropriate predictive biomarkers in the panel, the frequency of each biomarker in the respective patient population, measurement of all biomarkers in the panel with the necessary precision, the correlation of each biomarker to clinical outcome, the proportion of associations based on clinical evidence rather than preclinical or animal models and the guidance provided to the treating oncologistin support of selecting a treatment for an individual patient. The available profiling services differ in content for each of these factors and documented treatment outcomes are the most suitable overall measure of success incomparing different services. We reviewed published clinical studies and case series of patients who underwent molecular profiling using Caris Molecular Intelligence ${ }^{\circ}$ (534 patients) [8-18], FoundationOne $e^{\circ}(2,675$ patients) [19-44], $\operatorname{PCDx}^{\text {tix }}(168$ patients) [45], and academic approaches developed at leading academic institutions (5,576 patients) [46-54] (Table 1).

Clinical benefit was reported in a number of different ways. In the physician-led studies, disease stabilization and improvement of profiling-guided outcomes compared to prior outcomes were considered to define clinical benefit while more conventional methods of assessing response were generally used in the academic studies. The clinical benefit in those patients in whom a treatment was selected based upon the molecular profile is summarized in Figure $1 \mathrm{~b}$. The benefit is highest with Caris Molecular Intelligence ${ }^{\oplus}(50 \%)$, followed by $\operatorname{PCDx}^{\mathrm{m}}(43 \%)$, FoundationOne ${ }^{\circ}(34 \%)$ and academic approaches (33\%). It was not possible to evaluate the clinical benefit of OncoDEEP ${ }^{\mathrm{m \omega}}$ as there has only been a single case report published to date [55].

\section{Clinical Utility}

Clinical utility is a measurement of both the influence that molecular profiling has on oncologist's treatment decisions and the clinical benefit of the resulting treatments. A high utility means treatment decisions for the majority of patients have been revised in line with the profiling results and that the chosen treatments led to improved clinical outcomes. The impact on treatment choice is directly dependent on the panel of biomarkers tested, the frequency of those biomarkers in the population and the level of evidence presented to the oncologist in support of a change in treatment decision.

In precision medicine in cancer, clinical utility is defined as the generation of clinical useful and relevant information that can change the course of the disease for a patient, resulting in improved outcomes 
Citation: Janssens J, Gallagher WM, Dean A, Ussia G, Stamp G (2017) Tumor Profiling-Directed Precision Cancer Therapy - Comparison of Commercial and Academic Clinical Utility. Int J Surg Surgical Porced 2: 123. doi: https://doi.org/10.15344/2456-4443/2017/123

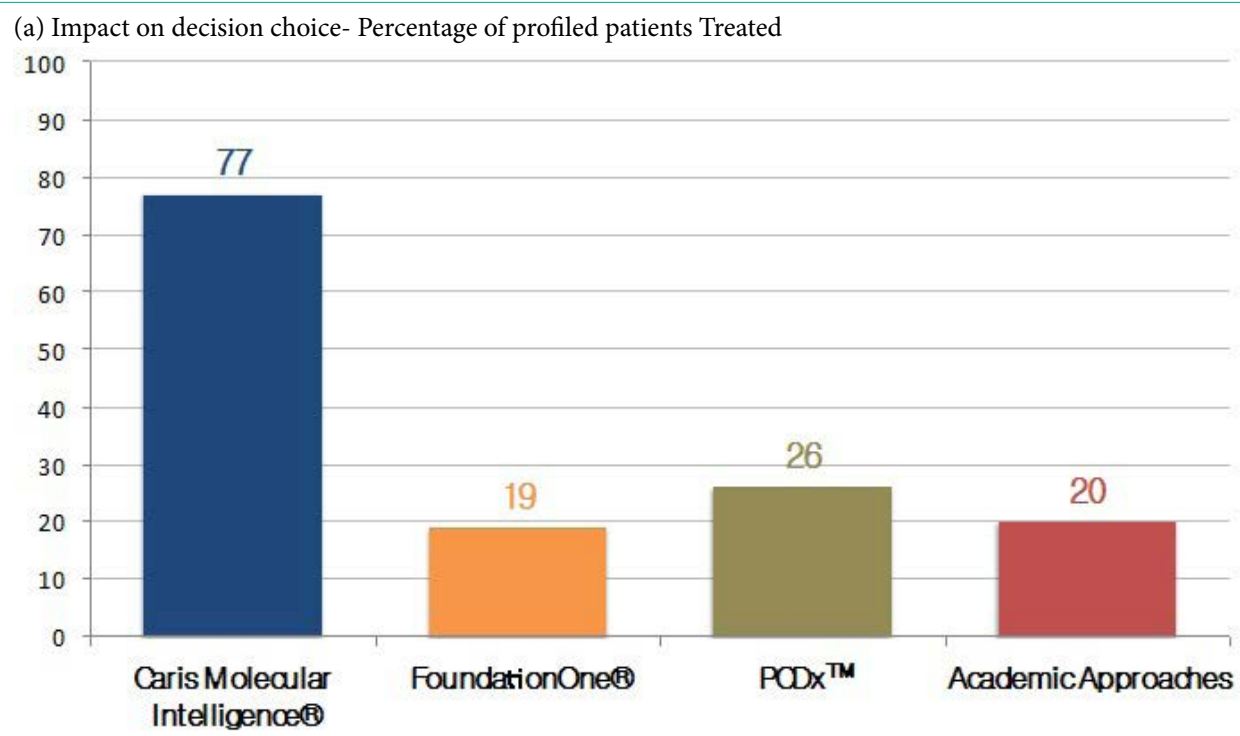

(b) Clinical Benefits: Percentage of treated \& evaluable patients with clinical benefits

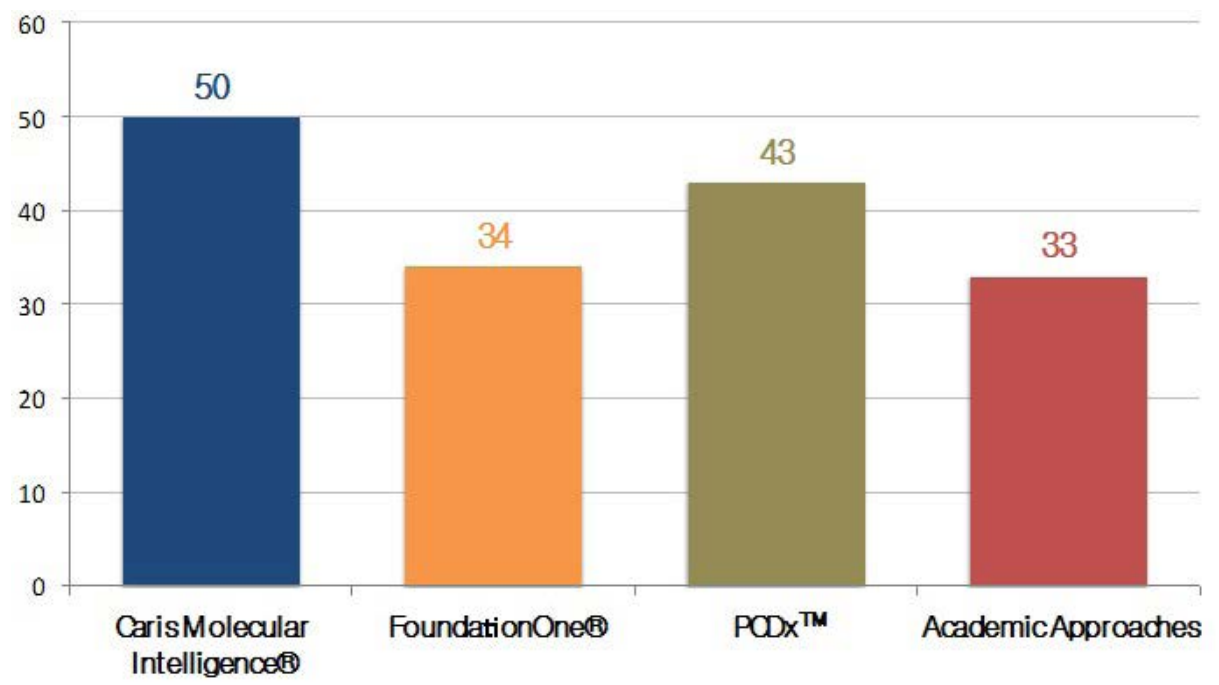

(c) Clinical Utility: Percentage of profiled patients with clinical benefit (ITT-like population)

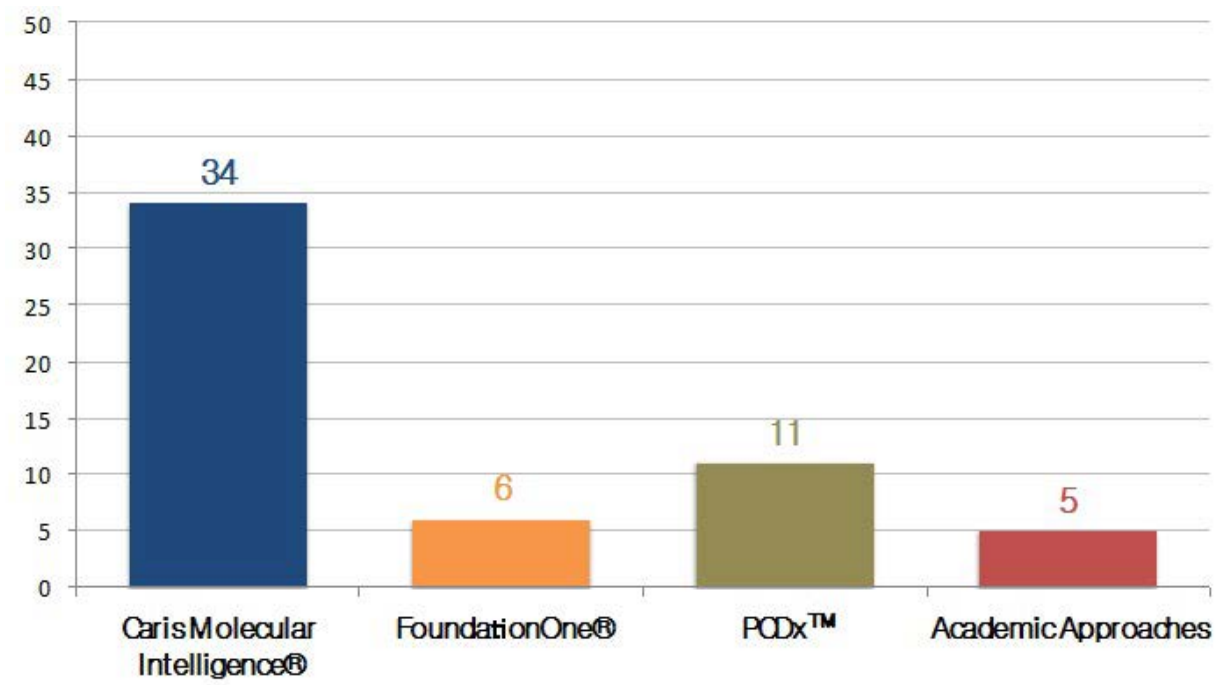

Figure 1: Comparison of impact on treatment choice (a), clinical benefit (b) and clinical utility (c) of academic and commercial profiling approaches. 
Citation: Janssens J, Gallagher WM, Dean A, Ussia G, Stamp G (2017) Tumor Profiling-Directed Precision Cancer Therapy - Comparison of Commercial and Academic Clinical Utility. Int J Surg Surgical Porced 2: 123. doi: https://doi.org/10.15344/2456-4443/2017/123

Page 4 of 10

\begin{tabular}{|c|c|c|c|c|c|c|c|c|c|c|}
\hline Author & Tumor Types & Technologies Used & $\begin{array}{l}\text { Patients } \\
\text { Profiled }\end{array}$ & \begin{tabular}{|l|} 
Patients \\
Treated
\end{tabular} & $\begin{array}{l}\text { \% Impact } \\
\text { on } \\
\text { Treatment } \\
\text { Choice }\end{array}$ & \begin{tabular}{|l|} 
Patients \\
evaluable \\
for Clinical \\
Benefit
\end{tabular} & $\begin{array}{l}\text { Measure of Clinical } \\
\text { Benefit }\end{array}$ & $\begin{array}{l}\text { Patients } \\
\text { with } \\
\text { Clinical } \\
\text { Benefit }\end{array}$ & \begin{tabular}{|l|} 
\% Clinical \\
Benefit \\
(compared \\
to Patients \\
Evaluable)
\end{tabular} & \begin{tabular}{|l} 
\% Clinical \\
Utility \\
(compared \\
to Patients \\
Profiled)
\end{tabular} \\
\hline \multicolumn{11}{|l|}{ Academic Approaches } \\
\hline Tsimberidou et al. . $^{46}$ & Refractory Solid Tumors & Ss & 1144 & 175 & 15 & 175 & RECIST Response & 47 & 27 & 4 \\
\hline Stockley et al. ${ }^{47}$ & Refractory Solid Tumors & NGS & 1640 & 245 & 5 & 84 & RECIST Response & 16 & 19 & 1 \\
\hline Le Tourneau et al..$^{48}$ & Refractory Solid Tumors & IHC, NGS & 496 & 195 & 39 & 99 & Disease control rate & 45 & 45 & 9 \\
\hline Massard et al. ${ }^{49}$ & Refractory Solid Tumors & $\begin{array}{l}\text { CGH, NGS, WES, } \\
\text { RNAseq } \\
\end{array}$ & 639 & 141 & 22 & 141 & RECIST Response & 47 & 33 & 7 \\
\hline Andrè et al..$^{50}$ & Refractory Breast Cancers & CGH, SS & 297 & 48 & 16 & 43 & RECIST Response & 13 & 30 & 4 \\
\hline Cousin et al. ${ }^{51}$ & Refractory Solid Tumors & NGS, CGH & 568 & 86 & 15 & 65 & Disease control rate & 31 & 48 & 5 \\
\hline Dienstmann et al..$^{52}$ & Refractory Colorectal Cancers & IHC, SS & 254 & 68 & 27 & 68 & CR/PR SD > 4 months & 11 & 16 & 4 \\
\hline Kim et a...53 & Refractory Colorectal Cancers & NGS, CNV & 407 & 103 & 25 & 103 & RECIST Response & 44 & 43 & \\
\hline Tanabe et al..$^{54}$ & Refractory Solid Tumors & NGS & 131 & 29 & 7 & 9 & RECIST Response & 3 & 33 & 2 \\
\hline TOTAL & & & 5576 & 1090 & 20 & 787 & & 257 & 33 & 5 \\
\hline \multicolumn{11}{|l|}{ Commercial Approaches } \\
\hline \multicolumn{11}{|l|}{ Caris Molecular Intelligence ${ }^{\circ}$} \\
\hline Von Hoff et al. ${ }^{8}$ & Refractory Solid Tumors & IHC, ISH, DNAMA, SS & 84 & 66 & 79 & 66 & PFS Ratio $\geq 1.3$ & 18 & 27 & 21 \\
\hline Jameson et al. ${ }^{9}$ & Refractory Breast Cancer & IHC, ISH, DNAMA, SS & 28 & 25 & 89 & 25 & PFS Ratio $\geq 1.3$ & 13 & 52 & 46 \\
\hline Epelbaum et al. ${ }^{10}$ & Refractory Pancreatic Cancer & IHC, ISH, DNAMA, SS & 55 & 30 & 55 & 24 & PFS Ratio $\geq 1.3$ & 9 & 38 & 16 \\
\hline Dean et al. ${ }^{11}$ & Refractory Solid Tumors & $\begin{array}{l}\text { IHC, ISH, DNAMA, } \\
\text { SS, NGS }\end{array}$ & 54 & 54 & 100 & 37 & PFS Ratio $\geq 1.3$ & 22 & 60 & 41 \\
\hline Popovtzer et al. ${ }^{12}$ & $\begin{array}{l}\text { Metastatic Adenoid Cystic } \\
\text { Carcinoma }\end{array}$ & IHC, ISH, DNAMA, SS & 14 & 11 & 79 & 11 & CR/PR/SD $>6$ months & 8 & 73 & 57 \\
\hline Ramanathan et al..$^{13}$ & Refractory Pancreatic Cancers & IHC, ISH, DNAMA, SS & 49 & 35 & 71 & 34 & OS $>6$ months & 14 & 41 & 29 \\
\hline Purim et al..$^{14}$ & Refractory Gastric Cancers & IHC, ISH, DNAMA, SS & 46 & 28 & 61 & 24 & PFS Ratio $\geq 1.3$ & 7 & 29 & 15 \\
\hline Seeber et al. ${ }^{15}$ & Refractory Solid Tumors & IHC, ISH, NGS & 58 & 40 & 69 & 40 & PFS Ratio $\geq 1.3$ & 22 & 55 & 38 \\
\hline El Ahmadie et al. ${ }^{16}$ & Refractory Solid Tumors & IHC, ISH, NGS & 73 & 69 & 95 & 59 & CR/PR/SD & 41 & 69 & 56 \\
\hline Chahine et al. ${ }^{17}$ & Refractory Solid Tumors & IHC, ISH, NGS & 34 & 27 & 79 & 23 & PFS Ratio $\geq 1.0$ & 15 & 65 & 44 \\
\hline El Nahas et al. ${ }^{18}$ & Refractory Solid Tumors & IHC, ISH, NGS & 39 & 26 & 67 & 22 & CR/PR/SD & 15 & 68 & 38 \\
\hline TOTAL & & & 534 & 411 & 77 & 365 & & 184 & 50 & 34 \\
\hline \multicolumn{11}{|l|}{ FoundationOne $^{\circ}$} \\
\hline Ferreira et al. ${ }^{19}$ & Refractory Solid Tumors & NGS & 66 & 11 & 17 & 11 & $\mathrm{CR} / \mathrm{PR} / \mathrm{SD}>6$ months & 3 & 27 & 5 \\
\hline Ganesan et al. ${ }^{20}$ & Refractory Breast Cancers & NGS & 106 & 16 & 15 & 16 & CR/PR/SD >6 months & 5 & 31 & 5 \\
\hline Brandao Moreira et al..21 & Refractory Breast Cancers & NGS & 20 & 9 & 45 & 9 & Objective response & 7 & & \\
\hline Brandao Moreira et a a..$^{22}$ & $\begin{array}{l}\text { Refractory Gastrointestinal } \\
\text { Cancers }\end{array}$ & NGS & 32 & 6 & 19 & 6 & Objective response & 4 & & \\
\hline Wheler et a a.. ${ }^{23}$ & Refractory Solid Tumors & NGS & 500 & 110 & 22 & 110 & $\mathrm{CR} / \mathrm{PR} / \mathrm{SD}>6$ months & 18 & 16 & 5 \\
\hline Schwaederle et al..$^{24}$ & Refractory Solid Tumors & NGS & 347 & 74 & 25 & 74 & CR/PR/SD >6 months & 27 & 36 & 8 \\
\hline Sohal et al. ${ }^{25}$ & Refractory Solid Tumors & NGS & 223 & 24 & 11 & 24 & CR/PR/SD >6 months & 7 & 29 & 3 \\
\hline Blumenthal et al. ${ }^{26}$ & Refractory Glioblastoma & NGS & 43 & 13 & 30 & 13 & RECIST Response & 0 & 0 & 0 \\
\hline Rodriguez-Rodriguez et al. ${ }^{27}$ & Gynecological Cancers & NGS & 69 & 25 & 36 & 23 & $\mathrm{CR} / \mathrm{PR} / \mathrm{SD} / \mathrm{CB}$ & 16 & 70 & 23 \\
\hline Johnson et al. ${ }^{28}$ & Refractory Solid Tumors & NGS & 103 & 18 & 17 & 18 & CR/PR/SD>6 months & 6 & 33 & 6 \\
\hline Hirschfield et al. ${ }^{29}$ & Refractory Solid Tumors & NGS & 100 & 31 & 31 & 31 & CR/PR/SD >6 months & 7 & 23 & 7 \\
\hline Groisberg et al..$^{30}$ & Sarcomas & NGS & 102 & 16 & 16 & 16 & CR/PR/SD >6 months & 8 & 50 & 8 \\
\hline Koenig et a $1 .{ }^{31}$ & $\begin{array}{l}\text { Refractory Gastrointestinal } \\
\text { Malignancies }\end{array}$ & NGS & 271 & 14 & 5 & 14 & Disease Control Rate & 3 & 21 & 1 \\
\hline Dhir et al. ${ }^{32}$ & Refractory Solid Cancers & NGS & 97 & 13 & 13 & 13 & $\mathrm{CR} / \mathrm{PR} / \mathrm{SD}>6$ months & 7 & 54 & 7 \\
\hline Yuan et al. ${ }^{.33}$ & Refractory Breast Cancers & NGS & 44 & 23 & 52 & 16 & $\mathrm{CR} / \mathrm{PR} / \mathrm{SD}>6$ months & 7 & 44 & 16 \\
\hline Grenader et al..$^{34}$ & Refractory Solid Cancers & NGS & 30 & 10 & 33 & 10 & $\mathrm{CR} / \mathrm{PR} / \mathrm{SD}>6$ months & 3 & 30 & 10 \\
\hline Gunderson et a..$^{35}$ & Gynecological Cancers & NGS & 62 & 6 & 4 & 4 & $\mathrm{CR} / \mathrm{PR} / \mathrm{SD}>6$ months & 2 & 50 & 3 \\
\hline Groisberg et al. ${ }^{36}$ & Rare Cancers & NGS & 95 & 9 & 9 & 9 & Disease Control rate & 7 & 78 & 7 \\
\hline Dalton et al. ${ }^{37}$ & Refractory Solid Cancers & NGS & 155 & 24 & 15 & 24 & CR/PR/SD >6 months & 6 & 25 & 4 \\
\hline Sicklick et al. ${ }^{38}$ & Treatment-naïve Cancers & NGS & 47 & 17 & 36 & 17 & CR/PR/SD >6 months & 9 & 53 & 19 \\
\hline Hodeib et al. ${ }^{39}$ & Rare Gynecological Cancers & NGS & 22 & 1 & 5 & 1 & Clinical response & 1 & 100 & 5 \\
\hline Bryce et al. ${ }^{40}$ & Refractory Solid Cancers & NGS & 141 & 29 & 21 & 29 & Clinical response & 13 & 45 & 9 \\
\hline TOTAL & & & 2675 & 499 & 19 & 488 & & 166 & 34 & 6 \\
\hline \multicolumn{11}{|l|}{ PCDx $^{\prime \prime}$} \\
\hline Radovich et al. ${ }^{45}$ & Refractory Solid Tumors & IHC, NGS & 168 & 44 & 26 & 44 & PFS Ratio $\geq 1.3$ & 19 & 43 & 11 \\
\hline TOTAL & & & 168 & 44 & 26 & 44 & & 19 & 43 & 11 \\
\hline
\end{tabular}

Table 1: Summary of Academic and Commercial Studies of Precision Medicine in Oncology.

CGH - Comparative genomic hybridization; IHC - Immunohistochemistry; NGS - Next generation sequencing; DNAMA - DNA Microarray, SS - Sanger Sequencing; WES - Whole exome sequencing RECIST - Response Evaluation Criteria in Solid Tumors; PFS - Progression Free Survival; CR - Complete Response; PR - Partial Response; SD - Stable disease; CB - Clinical benefit 
Citation: Janssens J, Gallagher WM, Dean A, Ussia G, Stamp G (2017) Tumor Profiling-Directed Precision Cancer Therapy - Comparison of Commercial and Academic Clinical Utility. Int J Surg Surgical Porced 2: 123. doi: https://doi.org/10.15344/2456-4443/2017/123

Page 5 of 10

[56 - 58]. The individual patient's likelihood of clinical benefit within the whole profiled patient group is the most critical measure for a patient and their oncologist when setting expectations of what a molecular profile can offer. Furthermore, clear communication of this information leads to realistic expectations and can build the patient's confidence and mental strength which in themselves are reported to lead to improved treatment outcomes in patients with metastatic solid tumors [59], a perhaps unexpected significant benefit of undertaking molecular profiling. If clinical benefit is restricted to a limited subset of patients within a tumor group (e.g. those with a specific mutation or only one 'targetable anomaly'), the expectations of successful outcomes for an individual patient should be similarly reduced. Based on the demonstrated impact on decision choice and the clinical benefit observed in guided treatments, Caris Molecular Intelligence ${ }^{\circ}$ shows clinical utility in $34 \%$ of all patients profiled, $\mathrm{PCDx}^{\mathrm{xa}}$ in $11 \%$, and FoundationOne $e^{\circ}$ and academic studies only in $6 \%$ and $5 \%$ respectively (Figure 1c). A representative graph comparing decision choice impact and clinical benefit from all approaches is shown in Figure 2.

There is a significant difference in clinical utility between Caris Molecular Intelligence ${ }^{\bowtie}$ and other commercial providers. In order to determine the underlying reason for this difference, the actual therapies chosen to treat individual patients that were reported in some of the clinical studies was analyzed (Table 2). It is apparent that after profiling with Caris Molecular Intelligence ${ }^{\star}$ a large number of patients were treated with cytotoxic chemotherapies (alone or in combination) whereas the fraction of targeted therapies was highest for FoundationOne ${ }^{\circ}$. This can be explained with the panel of biomarkers used in the respective molecular profiling, since FoundationOne ${ }^{\bullet}$ is limited to genomic profiling. The same biomarkers would also be measured with Caris Molecular Intelligence ${ }^{\oplus}$ and in addition, Caris
Molecular Intelligence ${ }^{ø}$ offers a number of biomarkers measured with IHC and ISH which are predictive of outcomes from treatment with cytotoxic chemotherapies. The information provided by NGS is mainly relevant to novel targeted drugs and many of such known targets are uncommon. In contrast, the protein biomarkers measured with Caris Molecular Intelligence ${ }^{\bullet}$ in addition are usually more frequently informative, leading to a higher clinical utility (more treatment decisions can be based on the results) and a higher clinical benefit. Based on the sparsity of relevant targets and a lack of availability of drugs targeting appropriate alterations, the clinical utility of comprehensive genomic profiling is limited to about $5 \%$ of all tumors profiled.

\section{Technical details, quality and turnaround time}

Different technical details such as the technology platforms used, the biomarker panels, attention to pre-analytical quality of tissues and original diagnosis, and the validation of individual assays can explain the differences in clinical utility in the profiled patients from the services we compared. Caris Molecular Intelligence ${ }^{\infty}$ has in-house pathological analysis and review and any issues regarding diagnosis and sample selection can be discussed among experts before committing to the full profiling.

With regard to the choice of NGS, Illumina is viewed as the industry standard, and has achieved FDA approval for medical use e.g. in diagnosing cystic fibrosis. Moreover the leading Academic institutions have chosen it (e.g. MSKCC, Dana Farber). An overview of the development and validation of a clinical NGS test is given by Frampton and his team [60]. Further guidance on the best practice and necessary steps for integration of NGS in clinical diagnostic molecular pathology laboratories has been described by the IQN Path

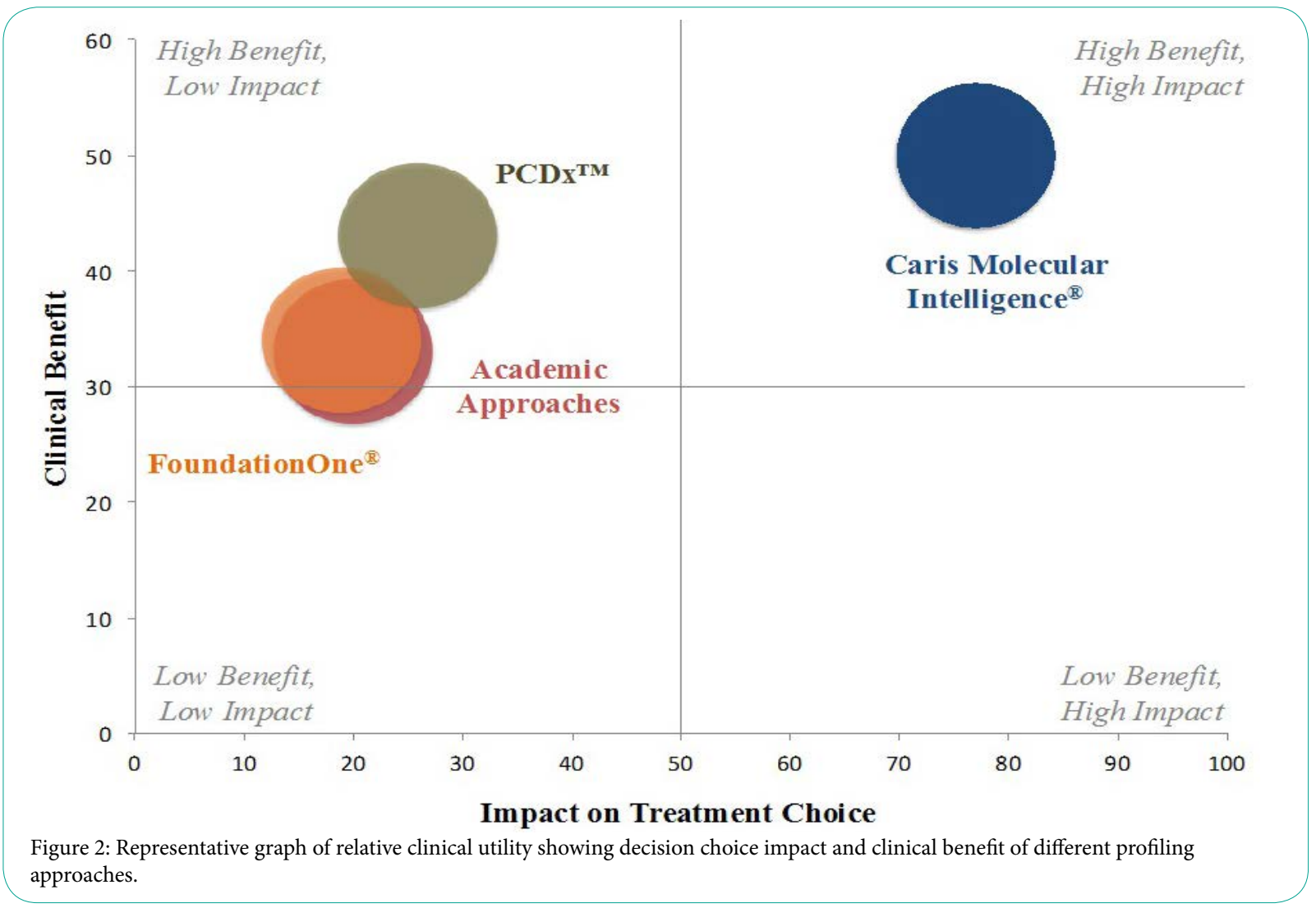

Int J Surg Surgical Porced 
Citation: Janssens J, Gallagher WM, Dean A, Ussia G, Stamp G (2017) Tumor Profiling-Directed Precision Cancer Therapy - Comparison of Commercial and Academic Clinical Utility. Int J Surg Surgical Porced 2: 123. doi: https://doi.org/10.15344/2456-4443/2017/123

Page 6 of 10

\begin{tabular}{|c|c|c|c|c|c|c|}
\hline \multirow[t]{2}{*}{ Profiling Approach } & \multicolumn{2}{|c|}{$\begin{array}{c}\text { Caris Molecular } \\
\text { Intelligence } \\
\text { 8-18 }\end{array}$} & \multicolumn{2}{|c|}{$\begin{array}{c}\text { FoundationOne } \\
20,24,25,26,27,29,30,31,32,33,34,37,40,41,42\end{array}$} & \multicolumn{2}{|c|}{$\mathrm{PCDx}^{\mathrm{ni4} 45}$} \\
\hline & $\mathrm{n}$ & $(\%)$ & $\mathrm{n}$ & $(\%)$ & $\mathrm{n}$ & $(\%)$ \\
\hline Overall Number of Patients & 385 & - & 302 & - & 19 & - \\
\hline Chemotherapy alone & 271 & $(70)$ & 5 & (2) & 2 & $(11)$ \\
\hline Chemotherapy plus hormone therapy & 5 & $(1)$ & 0 & - & 0 & - \\
\hline Chemotherapy plus targeted therapy & 63 & $(16)$ & 24 & (8) & 3 & $(16)$ \\
\hline Targeted Therapy alone & 30 & (8) & 202 & $(67)$ & 9 & $(47)$ \\
\hline Hormone therapy alone & 12 & (3) & 2 & $(<1)$ & 0 & - \\
\hline Targeted therapy plus hormone therapy & 3 & $(<1)$ & 11 & (4) & 0 & - \\
\hline Immunotherapy alone & 1 & $(<1)$ & 6 & $(2)$ & 2 & $(11)$ \\
\hline Investigational agents & 0 & - & 52 & $(17)$ & 3 & $(16)$ \\
\hline Monotherapy & 143 & $(37)$ & 225 & $(75)$ & 13 & $(68)$ \\
\hline Combination therapy & 242 & $(63)$ & 77 & $(25)$ & 6 & $(32)$ \\
\hline
\end{tabular}

Table 2: Breakdown of treatments used in different commercial profiling approaches.

ASBL group [61]. Caris Molecular Intelligence ${ }^{\oplus}$ and FoundationOne ${ }^{\star}$ use Illumina but $\mathrm{PCDx}^{\mathrm{m}}$ and OncoDEEP ${ }^{\mathrm{m}}$ use Ion Torrent which has not been approved for medical diagnostics and is intended for academic research. This is related to the fact that only hotspots on a single strand of DNA are sequenced in only one direction, which leads to a high error rate, in particular for homologous insertions and deletions where the error rate is doubled compared to Illumina[62]. The Ion Torrent platform does have advantages in turnaround time due to the shorter time needed to sequence data from hotspots only as shown in a direct comparison of the $\mathrm{PCDx}^{\mathrm{Tm}}$ and FoundationOne ${ }^{\infty}$ platforms when the median turnaround time was 9 days earlier for $\mathrm{PCDx}^{\text {tw }}$ compared to FoundationOne ${ }^{\oplus}$ [63]. The multiplatform approach of $\mathrm{PCDx}^{\mathrm{Tn}}$ also revealed more associations with commercially available drugs and less clinical trial associations than the single platform approach of FoundationOne ${ }^{\oplus}$. A recent case report highlights the discordance between the two NGS platforms and the possible challenges in managing a patient resulting from it [64]. Reliable detection of sensitizing mutations associated with commercially available drugs is critical and a major problem is that many of the platforms may lack rigorous analytic validation. For example, a comparison of EGFR activating mutations sensitive to erlotinib or gefitinib between FoundationOne ${ }^{\oplus}$ and local testing found that 12 of $71(17 \%)$ cases identified with FoundationOne ${ }^{\oplus}$ were missed by prior local testing [65].

The different companies also vary in their approach they take towards protein testing using IHC. Ideally each IHC test must adhere to the strict quality requirements from CAP, CLIA and NYS$\mathrm{DOH}$ and meticulous testing of all reagents for use in assays would be mandatory as described by O'Hurley and colleagues [66]. Caris Life Sciences has developed laboratory-developed tests (LDT) with comprehensive testing of all reagents used for its entire panel of IHCs (except where an IVD test kit is commercially available).While the NGS-section of the OncoDNA laboratory is ISO15189 accredited all other tests are performed without meeting internationally accepted quality standards for clinical use. OncoDEEP ${ }^{\text {mix }}$ has also included phosphorylated proteins in their panel but the phosphorylation of proteins is known to be highly sensitive to pre-analytical processing producing results that may be misleading for a treatment decision [67].The technical details of the services are summarized in Table 3.
The turnaround time of commercially available profiling services is advertised between 4 and 14 working days depending on service provider; the time for sample shipment must be added to this. For a detailed comparison, please see Table 3 .

\section{Technical details, quality and turnaround time}

To date, the majority of traditional cost effectiveness models have been designed to show a cost benefit in adding one drug or regimen over an existing comparator. In looking at the cost effectiveness of precision medicine approaches, new models must be considered. Previous modeling work from Shen and colleagues based on the Cancer Genome Atlas (TCGA) estimated the cost of finding a theoretically actionable mutation at between $\$ 6,254$ and $\$ 22,907$ and a pharmaceutically actionable mutation at between $\$ 8,035$ and $\$ 55,556$ depending on tumor type [68]. The true cost of profiling should not be considered as a reflection of the unit price but rather the amount that needs to be invested to bring clinical benefit to a patient. The key factors that must be taken into consideration are how often a molecular profile leads to a guided treatment being given to a patient, how often that treatment leads to clinical benefit, and the overall clinical utility. If we assume that all drugs selected by profiling are of comparable benefit, then it is imperative that impact on decision choice must be used to differentiate between approaches as it gives the easiest estimate of return on investment, be it in individual patients or on a larger scale from medical care providers.

List prices for the respective services were based on telephone enquiry or accessed online [68,69]. A summary of the cost effectiveness of the commercial and academic approaches based on the unit price of testing and the clinical utility is presented in Table 4. It is clear that approaches that have low clinical utility in the overall profiled population are unsustainable. The cost of profiling per patient tested with clinical benefit using Caris Molecular Intelligence ${ }^{\varpi}$ at $\$ 19,118$ is less than a quarter of the equivalent cost of $\$ 96,667$ using FoundationOne ${ }^{\otimes}$ 's NGS only approach and half that of the other approaches. This cost does not include any consideration for the recommended therapies, which would further extend the gap in cost given the high price of targeted therapies compared to conventional cytotoxic agents. This also means that approaches that do not show 
Citation: Janssens J, Gallagher WM, Dean A, Ussia G, Stamp G (2017) Tumor Profiling-Directed Precision Cancer Therapy - Comparison of Commercial and Academic Clinical Utility. Int J Surg Surgical Porced 2: 123. doi: https://doi.org/10.15344/2456-4443/2017/123

Page 7 of 10

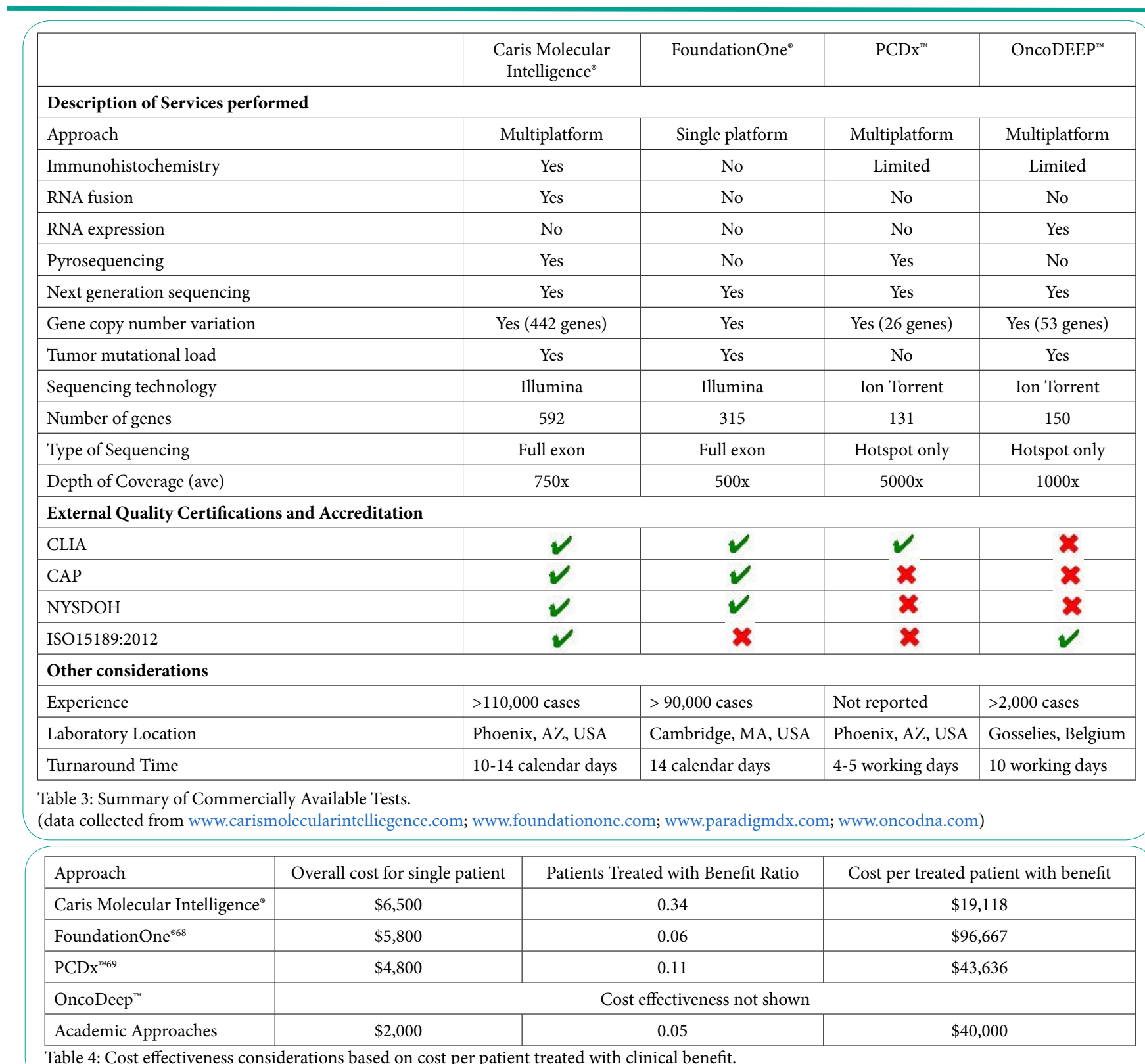

any clinical utility must be treated with some skepticism, as the cost effectiveness cannot be estimated. We estimated the true cost for an in-house approach that must include not only full costs (as opposed to the commonly adopted "marginal costs") of the materials and staff time, but also the ongoing investments in space, equipment, and ongoing training. The predicted number of patients profiled per year is also critical as a low throughput is likely not cost-effective.

\section{Conclusion}

Patients, medical care providers, pharmaceutical companies, researchers and oncologists have different requirements to utilize molecular tumor profiling for effectively treating advanced cancers. Patients demand a service that gives them a high likelihood of individual benefit (=clinical utility) at a justifiable price; often, they are not able or willing to pay excessive amounts for a molecular profiling with a low expectation of success. Medical care providers need to evaluate cost effectiveness of molecular profiling for the entire cohort of their insured patients, with a method that allows comparison with other innovations in health care. They need to provide care packages which include molecular profiling and are commercially viable and seek to avoid large expenditures on ineffective therapies.

A secondary advantage for pharmaceutical companies is the acceleration of clinical studies because patients with rare alterations can be reliably identified with molecular profiling and factored into clinical studies (perhaps explaining non-responders to a novel agent) or identifying specific cohorts for early development of therapies. Profiling data is also of high interest for the identification and selection of targets and the design of new drugs.

Oncologists need to navigate between all these needs and find solutions that satisfy all major stakeholders in the treatment of their patients. We showed that molecular profiling with NGS only is very 
Citation: Janssens J, Gallagher WM, Dean A, Ussia G, Stamp G (2017) Tumor Profiling-Directed Precision Cancer Therapy - Comparison of Commercial and Academic Clinical Utility. Int J Surg Surgical Porced 2: 123. doi: https://doi.org/10.15344/2456-4443/2017/123

Page 8 of 10

well suited to search for patients that meet the inclusion criteria for clinical trials of novel targeted drugs. It is therefore no surprise that academic institutions and drug companies mainly support broader use of NGS alone, be it through their in house developed profiling or through Foundation Medicine.

Molecular profiling reveals more insights; however, the information gained is often telling us about potential oncogenic drivers of the individual cancer rather than informing a treatment decision. When it comes to the selection of commercially available drugs, NGS has limited utility because most frequently proteins are identified as predictive biomarkers for conventional cytotoxic drugs, which remain the backbone of treatment in oncology. If the benefit for individual patients is the main goal it is relevant that the impact on decision choice of NGS is only around $20 \%$ and clinical utility is no higher than 5\%.Given the diversity between the biomarker panels and the quality and reproducibility of the tests offered, it is important that a molecular profiling service has a track record and provides solid evidence of proven clinical utility and benefit. This creates a basis for a clinical evaluation and comparison. Offerings with no or little clinical data cannot be recommended for use in the clinical setting(although they might be suitable for research evaluation).

In the US, every commercial laboratory needs to be certified by CAP and CLIA who provide stringent guidance and have instituted regular auditing procedures. Quality requirements are even higher in some states, for example in New York, where the Department of Health enforces strict criteria for reproducibility and constant results in the population over time. In Europe, quality has traditionally been demonstrated by ISO 15189 accreditation but is more focused on processes and not on the precision of specific laboratory tests or on pre-analytical requirement specifications. We have reviewed the quality thresholds met by Caris Life Sciences, Foundation Medicine, Paradigm, and OncoDNA. Only Caris Life Sciences and Foundation Medicine are allowed to offer their entire services in New York, Paradigmdx holds CLIA certification and OncoDNA is not even approved to sell in the US at all. In house services are only held accountable to local standards and only in the US one can safely assume that acceptable quality criteria are met. The new Diagnostics Regulation (IVDR), which was endorsed by the European Parliament on April 5, 2017, may address this issue in future [70].

The validation of IHC tests for which no standardized antibodies are commercially available is complex and comprises extensive testing of the reagents. Only very few academic institutions have research projects in which this is included and Caris Life Sciences is the only commercial laboratory that has implemented this approach to quality assurance. It also emphasizes the necessity of having inhouse expertise in pathology and analysis to corroborate the IHC findings with appropriate and validated controls. All these extra steps may simply not be economical for smaller companies and academic institutions, but the additional complexity is translating into better clinical outcomes and therefore well worth the expense.

When we compared the clinical utility achieved with the different platforms, it was impressive to see how uniform the results were between the offerings including NGS only. In a large number of patients, both FoundationOne ${ }^{\bullet}$ and NGS in-house testing has proven decision choice impact in about $20 \%$ of the patients and brings clinical utility to $5 \%$ of the patients. This is no surprise as the frequency of driver mutations and the number of associated drugs is limited. Adding on more tests with different platforms, mainly IHC, adds more clinical utility. Both $\mathrm{PCDx}^{\mathrm{na}}$ and Caris Molecular Intelligence have included a number of IHCs in their offerings. PCDx ${ }^{\mathrm{m} m}$ achieves a twofold and Caris Molecular Intelligence ${ }^{\circ}$ achieves a six fold higher clinical utility rate. Caris Molecular Intelligence ${ }^{\infty}$ has the highest clinical utility and benefit because it includes the most clinically relevant biomarkers.

The high clinical utility from Caris Molecular Intelligence ${ }^{\star}$ translates into the best cost-effectiveness. While both FoundationOne $e^{\oplus}$ and inhouse NGS profiling share the same clinical utility we assumed a lower cost for in-house testing, well aware that this may not include the true cost of the testing. Under these assumptions, FoundationOne would be the most expensive approach, in-house testing and $\mathrm{PCDx}^{\mathrm{Tw}}$ would cost half of that, and Caris Molecular Intelligence ${ }^{\circ}$ could be delivered at a quarter of the costs. A detailed analysis of the cost effectiveness is not only essential for medical care providers but also every patient who needs to pay for their profiling should be informed of these aspects by the treating physician. A very important but non-measurable benefit from a high clinical decision impact is the level of confidence for patients and their treating oncologists in having chosen a drug with a higher likelihood of benefit than a randomly selected drug.

In conclusion, molecular profiling has become an essential element in the selection of treatments for advanced or metastatic solid tumors.

\section{Competing Interests}

WMG is a co-founder of and Chief Scientific Officer with OncoMark Limited. The other authors have no relevant affiliations or financial involvement with any organization or entity with a financial interest in or financial conflict with the subject matter or materials discussed in the manuscript.

\section{Funding}

WMG is supported by the Irish Cancer Society Collaborative Cancer Research Centre BREAST-PREDICT (CCRC13GAL) and the Science Foundation Ireland Investigator Programme OPTi-PREDICT (grantcode 15/IA/3104). The other authors received no funding for this work

\section{References}

1. Meric-Bernstam F, Brusco L, Shaw K, Horombe C, Kopetz S, et al. (2015) Feasability of large-scale genomic testing to facilitate enrollment onto genomically matched clinical trials. J ClinOncol 33: 2753-2762.

2. Zehir A, Benayed R, Shah RH, Syed A, Middha S, et al. (2017) Mutational landscape of metastatic cancer revealed from prospective clinical sequencing of 10,000 patients. Nat Med 23: 703-713.

3. Prasad V (2016) Perspective: The precision-oncology illusion. Nature 537: S63.

4. Abrahams E, Eck S (2016) Molecular medicine: Precision oncology is not an illusion. Nature 539: 357 .

5. Schram AM, Berger MF and Hyman DM (2017) Precision oncology: Charting a path forward to broader deployment of genomic profiling. PLoS Med 14: e1002242.

6. Aggarwal A, Fojo T, Chamberlain C, Davis C, Sullivan R, et al. (2017) Do patient access schemes for high-cost cancer drugs deliver value to society? - Lessons from the NHS Cancer Drugs Fund. Ann Oncol Ann Oncol 28:1738-1750

7. Turck R (2017) Oncology drug costs - the imaginary crisis? Ann Oncol 28 427-431.

8. Von Hoff DD, Stephenson JJ Jr, Rosen P, Loesch DM, Borad MJ, et al. ((2010) Pilot study using molecular profiling of patients' tumours to find potential targets and select treatments for their refractory cancers. J Clin Oncol 28: 4877-4883 
Citation: Janssens J, Gallagher WM, Dean A, Ussia G, Stamp G (2017) Tumor Profiling-Directed Precision Cancer Therapy - Comparison of Commercial and Academic Clinical Utility. Int J Surg Surgical Porced 2: 123. doi: https://doi.org/10.15344/2456-4443/2017/123

Page 9 of 10

9. Jameson GS, Petricoin EF, Sachdev J, Liotta LA, Loesch DM, et al. (2014) A Pilot Study Utilizing Multi-omic Molecular Profiling to find Potentia Targets and Select Individualized Treatments for Patients with Previously Treated Metastatic Breast Cancer. Breast Cancer Res Treat 147: 579-588.

10. Dean A, Wallace R (2016) Clinical outcomes of patients with rare and heavily pretreated solid tumors treated according to the results of tumor molecular profiling. BioMed Res Int 2016: 4627214.

11. Popovtzer A, Sarfaty M, Limon D, Marshack G, Perlow E, et al. ( 2015) Metastatic Salivary Gland Tumours: A Single-center Study Demonstrating the Feasibility and Potential Clinical Benefit of Molecular-profiling-guided Therapy. BioMed Res Int 2015: 614845

12. Barrett MT, Lenkiewicz E, Evers L, Holley T, Aziz M, Kiefer J, et al. (2012) Abstract 3697: Phase II study of therapy selected by molecular profiling in patients with previously treated metastatic pancreatic cancer - SU2C-001. Cancer Res 72: Abstract nr 3697.

13. Epelbaum R, Shacham-Shmueli E, Klein B, Agbarya A, Brenner B, et al (2015) Molecular Profiling-Selected Therapy for Treatment of Advanced Pancreaticobiliary Cancer: A Retrospective Multicenter Study. BioMed Res Int 2015: 681653.

14. Purim O, Beny A, Inbar MJ, Brenner B, Dudnik E, et al. (2017) Molecular profiling-selected treatment in metastatic gastric and esophageal cancer: Real-life clinical experience. Journal of Clinical Oncology 35: e15537.

15. Seeber A, Gastl G, Ensinger C et al. Treatment of patients with refractory metastatic cancer according to molecular profiling on tumor tissue in the clinical routine: an interim-analysis of the ONCO-T-PROFILE project. Genes Cancer 7: 301-310.

16. Ahmadie A, El KarakF, Farhat F, Kattan J, Hanna C, et al. (2015) Molecula Profiling guided treatment in refractory solid tumours: practical impact and clinical responses: Experience of a single center. European Journal of Cancer 51: S43.

17. Chahine G, Nasr F, Saleh K (2016) Clinical outcomes of patients with previously treated advanced or metastatic cancer before or after tumour profiling: results of a monocentric pilot study in Lebanon, ICACT.

18. El Nahas T (2017) Multiplatform molecular profiling to determine treatment selection in rare, aggressive, refractory and metastatic cancers with limited treatment options, ICACT.

19. Ferreira AR, Gomes ASDA, Costa FP (2014) Next generation sequencing - A key in search of locks? Ann Oncol 25: iv560.

20. Ganesan P, Moulder S, Lee JJ, Janku F, Valero V, et al. (2014) Triplenegative breast cancer patients treated at MD Anderson Cancer Center in phase I trials: Improved outcomes with combination chemotherapy and targeted agents. Mol Cancer Ther 13: 3175-3184.

21. Brandao Moreira R, Peixoto R, de Sousa TTS, Cruz M, Maluf $F$, et al (2015) Next-generation sequencing (NGS) in metastatic breast cance (mBC) patients: Translation from sequence data into clinical practice. J Clin Oncol 33: 133-133.

22. Brandao Moreira R, Alessandretti MB, Abrahao CM, et al. (2015) Nextgeneration sequencing (NGS) in metastatic gastrointestinal cancer ( $\mathrm{mGIC}$ ) patients: Translation from sequence data into clinical practice. J Clin Onco 33: $133-133$

23. Wheler JJ, Janku F, Naing A, Li Y, Stephen B, et al. (2016) Cancer Therapy Directed by Comprehensive Genomic Profiling: A Single Center Study. Cancer Res 76: 3690-3701.

24. Schwaederle M, Parker BA, Schwab RB, Daniels GA, Piccioni DE, et al (2016) Precision oncology: the UC San Diego Moores Cancer Center PREDICT experience. Mol Cancer Ther 15: 743-752.

25. Sohal DP, Rini BI, Khorana AA, Dreicer R, Abraham J, et al. (2016) Prospective clinical study of precision oncology in solid tumours. J Natl Cancer Inst 108: pii-djv332.

26. Blumenthal DT, Dvir A, Lossos A, Tzuk-Shina T, Lior T, et al. Clinical utility and treatment outcome of comprehensive genomic profiling in high gradeglioma patients. J Neurooncol 130: 211-219.

27. Rodriguez-Rodriguez L, Hirshfield KM, Rojas V, DiPaola RS, Gibbon D, et al. (2016) Use of comprehensive genomic profiling to direct point-of-care management of patients with gynecologic cancers. Gynecol Oncol 141 $2-9$.

Int J Surg Surgical Porced

ISSN: 2456-4443
28. Johnson DB, Dahlman KH, Knol J, Gilbert J, Puzanov I, et al. (2014) Enabling a genetically informed approach to cancer medicine: A retrospective evaluation of the impact of comprehensive tumor profiling using a targeted next-generation sequencing panel. Oncologist 19: 616622

29. Hirshfield KM, Tolkunov D, Zhong $\mathrm{H}$, Ali SM, Stein MN, et al. Clinica actionability of comprehensive profiling for management of rare or refractory cancers. Oncologist 21: 1315-1325.

30. Groisberg R, Hong DS, Holla V, Janku F, Piha-Paul S, et al. (2017) Clinical genomic profiling to identify actionable alterations for investigational therapies in patients with diverse sarcomas. Oncotarget 8: 39254-39267.

31. Koenig KL, Burkart J, Mikhail S, Wu CSY, Noonan Am, et al. (2016) Therapeutic impact and timing of gastrointestinal malignancy genomic profiling: A single-institution experience. J Clin Oncol 34: 584-584.

32. Dhir M, Choudry HA, Holtman MP, Pingpank JF, Ahrendt SA, et al. (2017) Impact of genomic profiling on the treatment and outcomes of patients with advanced gastrointestinal malignancies. Cancer Med 6: 195-206.

33. Yuan Y, Yost SE, Yuan YC, Solomon NM, Mambetsariev I (2017) genomic mutation-driven metastatic breast cancer therapy: a single center experience. Oncotarget 8: 26414-26423.

34. Grenader T, Tauber R, Shavit $L$ (2016) Next-generation-sequencing in patients with advanced cancer: are we ready for widespread clinical use? A single institute's experience. Anticancer Drugs 27: 899-907.

35. Gunderson CC, Rowland MR, Wright DL, Andrade KL, et al. (2016) Initiation of a formalized precision medicine program in gynecological oncology. GynecolOncol 141: 24-28.

36. Groisberg R, Hong DS, Janku F, Groisberg R, Hong DS, Janku F, et al. (2017) Clinical next generation sequencing for precision medicine in rare cancers. J Clin Oncol 35: 2582-2582.

37. Dalton WB, Forde PM, Kang H, Connolly Rm, Stearns V, et al. (2017) Personalized medicine in the oncology clinic: Implementation and outcomes of the Johns Hopkins Molecular Tumor Board. JCO Precision Oncology 1: 1-19.

38. Hodeib M, Prendergast EN, Liu AY, Fahey JN, Elvin JA, et al. (2017) Comprehensive genomic profiling of rare gynecological malignancies. Gynecologic Oncology 145: 212

39. Sicklick JK, Leyland-Jones B, Kato S, Hahn M, Williams C, et al. (2017) Personalized, molecularly matched combination therapies for treatmentna. J Clin Oncol 35: 2512-2512.

40. Bryce AH, Egan JB, Borad MJ, Stewart AK, Nowakowski GS, et al. (2017) Experience with precision genomics and tumor board, indicates frequent target identification, but barriers to delivery. Oncotarget 8: 27145-27154.

41. Braiteh F, Sharman JP, Richards DA, Skelton MR, DeMarco C, et al. (2014) Clinical NGS-based cancer genomic profiling impacts physician treatment decisions in advanced solid tumours. J Clin Oncol 32: 11109-11109.

42. DiBardino DM, Saqi A, Elvin JA, Greenbowe J, Suh JH, et al. (2016) Yield and clinical utility of next-generation sequencing in selected patients with lung adenocarcinoma. Clin Lung Cancer 17: 517-522.

43. Mantripragada KC, Olszewski AJ, Schumacher A, Perez K, Birnbaum A, et al. (2016) Clinical Trial Accrual Targeting Genomic Alterations after NextGeneration Sequencing at a Non-National Cancer Institute-Designated Cancer Program. J Oncol Pract 12: e396-e404.

44. Reinbolt RE, Tolliver K, Abdel-Rasoul M, Timmers CD, Ramaswamy B, et al. (2016) Decision impact analysis of comprehensive genomic profiling (CGP) in advanced breast cancer: A prospective study. J Clin Oncol 34: 11578-11578.

45. Radovich M, Kiel PJ, Nance SM, Niland EE, Parsley ME, et al. (2016) Clinical benefit of a precision medicine based approach for guiding treatment of refractory cancers. Oncotarget 7: 56491-56500.

46. Tsimberidou AM, Iskander NG, Hong DS, Wheler JJ, Falchook GS, et al. (2012) Personalized medicine in a phase I clinical trials program: the MD Anderon Cancer Center initiative. Clin Cancer Res18: 6373-6383.

47. Stockley TL, Oza AM, Berman HK Leighl NB, Knox JJ, et al. (2016) Molecular profiling of advanced solid tumors and patient outcomes with genotype-matched clinical trials: The Princess Margaret IMPACT/ COMPACT trial. Genome Med 8: 109 . 
Citation: Janssens J, Gallagher WM, Dean A, Ussia G, Stamp G (2017) Tumor Profiling-Directed Precision Cancer Therapy - Comparison of Commercial and Academic Clinical Utility. Int J Surg Surgical Porced 2: 123. doi: https://doi.org/10.15344/2456-4443/2017/123

Page 10 of 10

48. Le Tourneau C, Delord JP, Gonçalves A, Gavoille C, Dubot C, et al. (2015) Molecularly targeted therapy based on tumour molecular profiling versus conventional therapy for advanced cancer (SHIVA): a multicenter, openlabel, proof-of-concept, randomized, controlled phase 2 trial. Lancet Oncology 16: 1324-1334.

49. Massard C (2015) Enriching phase I trials with molecular alterations: Interim analysis of 708 patients enrolled in the MOSCATO 01. Ann Oncol 26: ii4

50. Andrè F, Bachelot T, Commo F, Campone M, Arnedos M, et al. (2014) Comparative genomic hybridization array and DNA sequencing to direct treatment of metastatic breast cancer: a multicenter, prospective trial (SAFIR01/UNICANCER). The Lancet Oncology 15: 267-274.

51. Cousin S, Grellety T, Toulmonde M, Auzanneau C, Khalifa E, et al. (2017) Clinical impact of extensive molecular profiling in advanced cancer patients. J HematolOncol 10: 45

52. Dienstmann R1, Serpico D, Rodon J, Saura C, Macarulla T, et al. (2012) Molecular profiling of patients with colorectal cancer and matched targeted therapy in phase I clinical trials. Mol Cancer Ther 11: 2062-2071.

53. Kim ST, Lee J, Hong M, Park K, Park JO, et al. (2015) The NEXT-1 (Next generation $\mathrm{pErsonlizedtX}$ with mulTi-omics and preclinical model) trial: prospective molecular screening trial of metastatic solid cancer patients, a feasibility analysis. Oncotarget 6: 33358-33368.

54. Tanabe Y, Ichikawa H, Kohno T, Yoshida H, Kubo T, et al. (2016) Comprehensive screening of target molecules by next-generation sequencing in patients with malignant solid tumors: guiding entry into phase I clinical trials. Mol Cancer 15: 73

55. Ramírez de Olano A, Bellmunt J, Rodrigo A, Álvarez L, Terrádez A, et al. (2017) A case report demonstrating the potential clinical benefit of exhaustive molecular profiling in an aggressive muscle-invasive high-grade metastatic urothelial carcinoma. Case Rep Oncol 10: 493-500.

56. Smart A (2006) A multi-dimensional model of clinical utility. Int J Qual Health Care 18: 377-382.

57. Peabody JW, Shimkhada R, Tong KB, Zubiller MB (2014) New thinking on clinical utility: Hard lessons for molecular diagnostics. Am J Manag Care 20: 750-756.

58. Borrebaeck CAK (2017) Precision diagnostics: moving towards protein biomarker signatures of clinical utility in cancer. Nat Rev Cancer 17: 199204.

59. Batty GD, Russ TC, Stamatakis E, Kivimäki M, et al. (2017) Psychological distress in relation to site specific cancer mortality: pooling of unpublished data from 16 prospective cohort studies. BMJ 356: j108.

60. Frampton GM, Fichtenholtz A, Otto GA, Wang K, Downing SR, et al. (2013) Development and validation of a clinical cancer genomic profiling test based on massively parallel DNA sequencing. Nat Biotechnol 31: 1023 1031.

61. Deans ZC, Costa JL, Cree I, Dequeker E, Edsjö A, et al. (2017) Integration of next-generation sequencing in clinical diagnostic molecular pathology laboratories for analysis of solid tumours; an expert opinion on behalf og IQN Path ASBL. Virchows Arch 470: 5-20.

62. Salipante SJ, Kawashima T, Rosenthal C, Hoogestraat DR, Cummings A e et al. (2014) Performance Comparison of Illumina and lon Torrent Next-Generation Sequencing Platforms for 16S rRNA-Based Bacterial Community Profiling. Appl Environ Microbiol 80: 7583-7591.

63. Weiss GJ, Hoff BR, Whitehead RP, Sangal A, Penny RJ, et al. (2015) Evaluation and comparison of two commercially available targeted nextgeneration sequencing platforms to assist oncology decision making. Onco Targets Ther 8: 959-967.

64. Simon A, Kourie HR, Kerger J (2017) Is there still a role for cytotoxic chemotherapy after targeted therapy and immunotherapy in metastatic melanoma? A case report and literature review. Chin J Cancer 36: 10.

65. Schrock AB, Frampton GM, Herndon D, Greenbowe JR, Wang K, et al. (2016) Comprehensive Genomic Profiling Identifies Frequent Drug Sensitive EGFR Exon 19 Deletions in NSCLC Not Identified by Prior Molecular Testing. Clin Cancer Res 22: 3281-3285.

66. O'Hurley G, Sjöstedt E, Rahman A, Li B, Kampf C, et al. (2014) Garbage in, garbage out: A critical evaluation of strategies used for validation of immunohistochemical biomarkers. Mol Oncol 8: 783-798.

67. David KA, Juhl H(2015) Immunohistochemical Detection of Phosphoproteins and Cancer Pathways. In: Lin F., Prichard J. (eds) Handbook of Practical Immunohistochemistry. Springer, New York, NY.
68. Shen C, Meric-Bernstam F, Su X et al. (2016) Prevalence of actionable mutations and copy number alterations and the price of a genomic testing panel. Oncotarget 7: 71686-71695.

69. https://assets.contentful.com/vhribv12Imne/5BJHWaCe1Gwu8CaksomG S8/653f19c8657097e43fad4aabbc6495a4/Patient_Information_Guide_5. pdf

70. http://www.paradigmdx.com/wp-content/uploads/2014/08/ ParadigmPCDxFAQs4-2014.pdf

71. http://data.consilium.europa.eu/doc/document/PE-15-2017-INIT/en/pdf
Int J Surg Surgical Porced

ISSN: 2456-4443
IJSSP, an open access journal

Volume 2. 2017. 123 